\title{
Analysis of SAR distribution in human head of antenna used in wireless power transform based on magnetic resonance
}

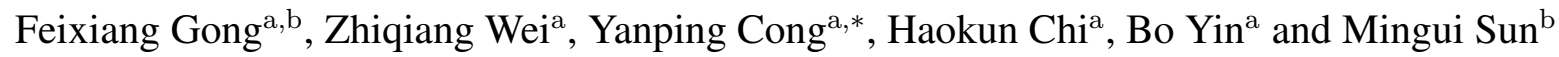 \\ ${ }^{a}$ School of Information Science and Engineering, Ocean University of China, Qingdao, Shandong, \\ China \\ ${ }^{\mathrm{b}}$ School of Medicine, University of Pittsburgh, Pittsburgh, PA, USA
}

\begin{abstract}
In this paper, a novel wireless power transfer antenna system was designed for human head implantable devices. The antenna system used the structure of three plates and four coils and operated at low frequencies to transfer power via near field. In order to verify the electromagnetic radiation safety on the human head, the electromagnetic intensity and specific absorption rate (SAR) were studied by finite-difference-time-domain (FDTD) method. A three-layer model of human head including skin, bone and brain tissues was constructed. The transmitting and receiving antenna were set outside and inside the model. The local and average SAR were simulated at the resonance frequency of $18.67 \mathrm{MHz}$ in two situations, in one scenario both transmitting and receiving coil worked, while in the other scenario only the transmitting coil worked. The results showed that the maximum of $10 \mathrm{~g} \mathrm{SAR}$ average value of human thoracic were $0.142 \mathrm{~W} / \mathrm{kg}$ and $0.148 \mathrm{~W} / \mathrm{kg}$, respectively, both were lower than the international safety standards for human body of the ICNIRP and FCC, which verified the safety of the human body in wireless power transmission based on magnetic coupling resonance.
\end{abstract}

Keywords: SAR (specific absorption rates), magnetic resonance, FDTD, WPT (wireless power transfer), four-layer

\section{Introduction}

Implantable medical devices are becoming hot spots for R\&D development of medical treatment. Implantable medical devices, such as heart pacemakers, artificial hearts, drug pumps, brain pacemakers play an important role in the field of human body health condition monitoring, treatment, maintenance, and rehabilitation. The head implantable device is a kind of biomedical equipment implanted into the brain with the purpose to stimulate brain tissue via electromagnetic signals. Apart from other cable medical devices, implantable medical devices generally use the high-energy battery to get the power supply. The useful time of the battery for head implantable device ranges from months to years. It greatly increases the economic burden of patients and the risk of secondary surgery when the battery is used up. The wireless transmissions of power become urgent needs in the development of the implantable medical device.

\footnotetext{
${ }^{*}$ Corresponding author: Yanping Cong, School of Information Science and Engineering, Ocean University of China, Qingdao, Shandong, China. Tel.: +86 18562630152; E-mail: congyp@163.com.
}

0928-7329/17/\$35.00 (C) 2017 - IOS Press and the authors. All rights reserved

This article is published online with Open Access and distributed under the terms of the Creative Commons Attribution NonCommercial License (CC-BY-NC 4.0). 
In the field of early medical implantable devices, the wireless energy transmission used ultrasonic or electromagnetic induction technology. However, these technologies could not satisfy the demand of long transmission distance and high transmission power at the same time. Highly efficient and reliable energy supply has become a key common bottleneck problem of human implantable devices. Resonance coupling power transmission technology is a new technology with wider applied range and low electromagnetic radiation, which can satisfy both the requirements of safety and electromagnetic compatibility [1]. It will also be greatly used in the field of medical and biological engineering for wireless power transfer to solve the uninterrupted power supply problem for implantable devices. Wireless power transmission technology via magnetic resonance works at low frequencies range $(1-20 \mathrm{MHz})$ which will produce electromagnetic radiation in near electromagnetic field. The absorption of electromagnetic energy of organism can cause biological effects. In order to verify the safety of the wireless power transmission technology brought by human implantable devices, it is necessary to research the distribution of electromagnetic radiation to head. An experimental study is restricted to measuring instruments and experimental environment. Numerical simulation of electromagnetic simulation technology can provide high-precision radiation model. It can better verify the radiation to the human head from implantable medical electronic devices with wireless energy transmission system [2]. The research on electromagnetic radiation into human body mostly use the SAR as the parameter [3]. Early studies of human radiation safety focused on wireless communication technology equipment (such as mobile phones) using the method of moments and FFT-CG iterative algorithm to analysis the electromagnetic radiation. Until 1990, Sullivan began to calculate the problem by using the method of finite-difference time-domain method (FDTD) [4]. There are more cases of studying the electromagnetic radiation to human body SAR by FDTD method, focusing on the interaction of small helical antenna, small PIFA antenna and dipole antenna [5]. Through these studies, the results of SAR calculation is related to the organizational structure of the human body, the location of the RF source [6,7]. Therefore, it's very important to obtain accurate results of the specific radiation for accurate simulation scene. Park studied electromagnetic radiation dose distribution and SAR of the human head model with a new kind of PIFA antenna [8]. Neubauer analyzed the operating frequency of the antenna and simulated the electromagnetic radiation to the human body when the phone works at $2450 \mathrm{MHz}, 1800 \mathrm{MHz}, 900 \mathrm{MHz}$ and 433 $\mathrm{MHz}$ [9]. Choi et al. used FDTD method to study the effects of electromagnetic radiation generated by mobile phones to human heads, they draw a three-dimensional distribution of magnetic and electric fields and the distribution curve of the specific absorption rate and established an effective method to reduce specific absorption rate [10]. Christ studied that radiation generated by near-field wireless power transmission (WPT) systems may pose a potential health hazard risk directly or indirectly by interfering with the medical implants. Advantages and disadvantages of numerical and experimental techniques are discussed on the implementation operating at $8 \mathrm{MHz}$ [11]. Xin emulates a real three-dimensional human body model and applies FDTD algorithm to calculate the SAR in different human tissues in the electromagnetic environment, where the transmit power is $25 \mathrm{~W}$ and the resonant frequency is $26615 \mathrm{kHz}$ [12]. $\mathrm{Xu}$ applied magnetic resonance technology to design a human implantable electronic device with small wireless charging systems. They used FDTD method to calculate the SAR distribution and electric field strength in electromagnetic environment generated by the implantable device around a one-layer head model. The results showed that the average SAR and the electromagnetic field strength into a head model were lower than the international safety standard limits [13]. Asma made a six-layer head model to evaluate SAR distribution to human brain which was generated by the dipole antenna. The E-field strength and SAR distribution in both one layer and six-layer human models were simulated which showed that the six-layer model was more close to reality situation [14]. 


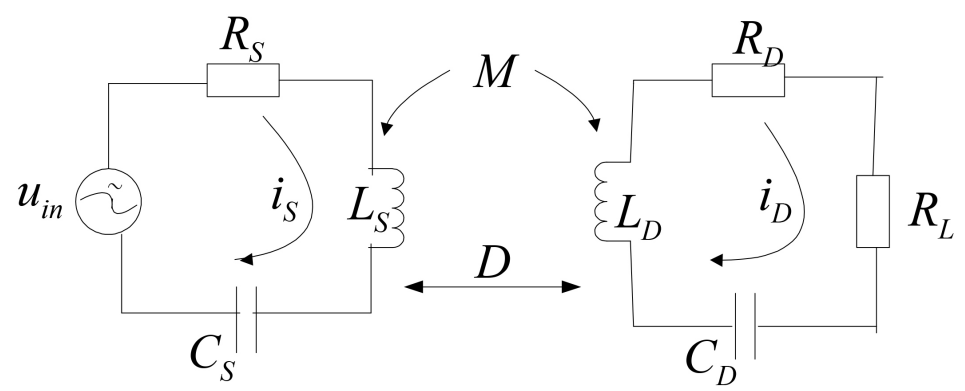

Fig. 1. Typical WPT system via magnetic resonance technology.
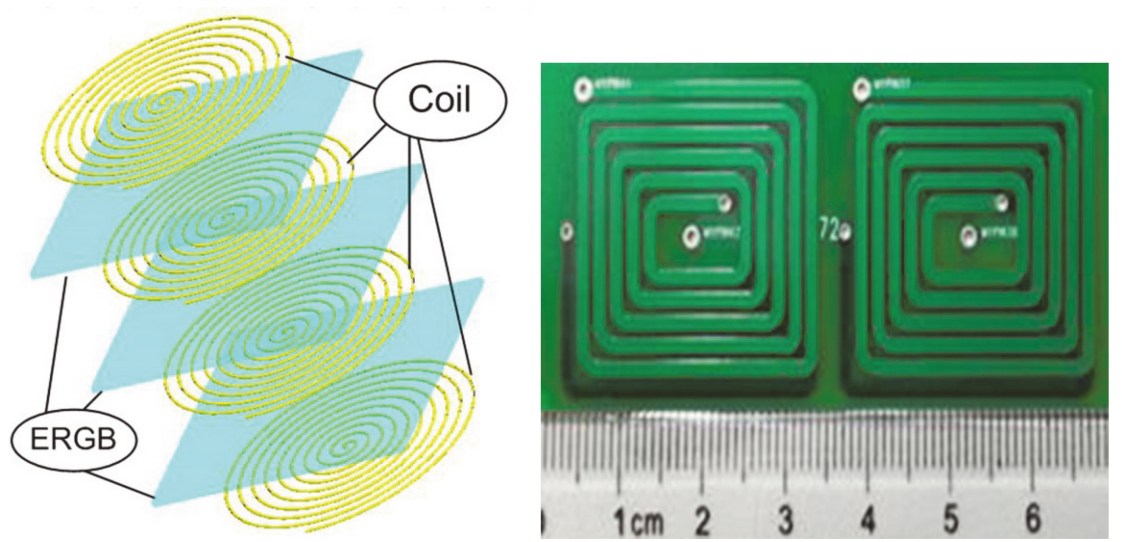

Fig. 2. Structure of four-layer coil used as resonance antenna.

In this paper, a small four-layer system of wireless power transfer antenna was designed for human head implantable devices, and the SAR and electromagnetic intensity were calculated by FDTD method. It was simulated by constructing a three-layer model approach for human head through HFSS software with the antennas inside and outside at a $6 \mathrm{~mm}$ distance. Local and average SAR were simulated at the resonance frequency in two situations, in one case both transmitting and receiving coil worked, while in the other case only the transmitting coil worked. The results showed that $10 \mathrm{~g}$ SAR average values of the head were $0.142 \mathrm{~W} / \mathrm{kg}$ and $0.148 \mathrm{~W} / \mathrm{kg}$, which were lower than the safety standards of the ICNIRP, which verified the safety of human head in WPT process based on the technology of magnetic resonance.

\section{System structure of wireless energy transmission via magnetic resonance}

WPT technology via magnetic resonance technology used two antennas which have the same Q-factor and resonance frequency. Most of the energy will be transferred efficiently through near field electromagnetic field via electromagnetic oscillation when the antennas system worked in the resonance state, as shown in Fig. 1.

The new technology has lower radiation, higher efficiency, longer transmission distance and satisfies both the requirements of the safety and electromagnetic compatibility. Therefore, it is gaining more and more attention due to its advantages in the field of medical and biological engineering [15], especially in human head implantable devices . 


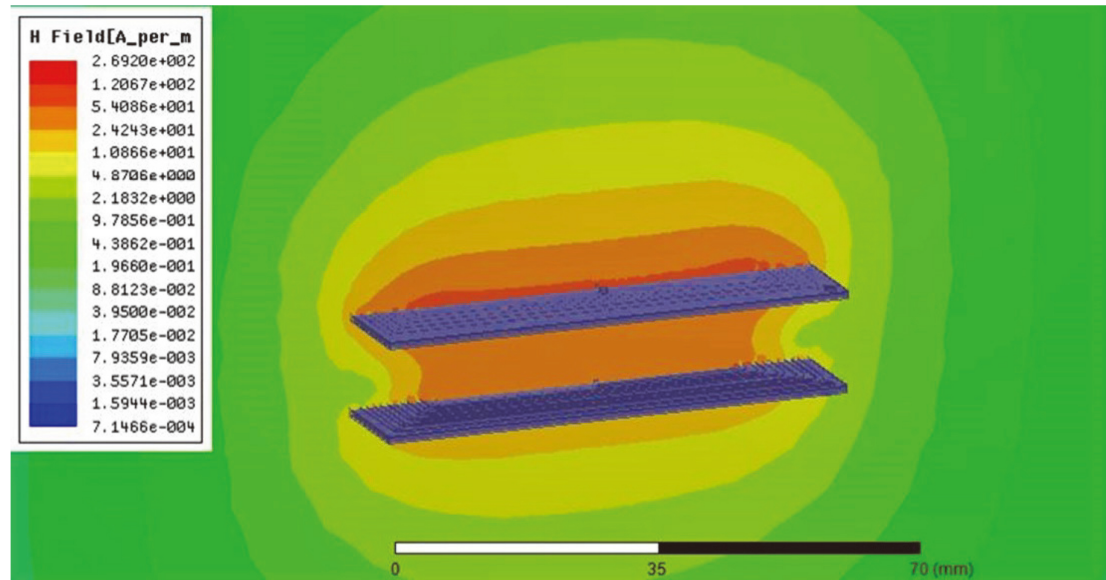

Fig. 3. Magnetic distribution of close field in resonance state.

\section{Design of four-layer antenna system}

The approach for our wireless power transmission implantable antenna is a four-layer design. The antenna is fabricated into four epoxy resin glass fabrics (ERGF) layers and the copper coils were set in the space between each layer as shown in Fig. 2. Therefore, the resonance frequency and Q factor can be adjusted by the distributed capacitance generated multilayer structure the thickness of each ERGF is $2 \mathrm{~mm}$, length is $35 \mathrm{~mm}$, width is $35 \mathrm{~mm}$, material is copper, width of the copper is $1 \mathrm{~mm}$, thickness of $\mathrm{t}$ copper is $0.035 \mathrm{~mm}$. Parameters of the antennas were simulated by FEM method through ANSOFT HFSS software. Resonance frequency is $18.67 \mathrm{MHz}$, and the Q factor is 62.23 . When antenna system works in a resonance state, energy will be transferred efficiently to the receiving antenna. The simulation result was shown in Fig. 3, which demonstrates that most of the energy were transferred from the transmitting coil to the receiving coil.

\section{Evaluation of SAR distribution in human head}

\subsection{Calculation of SAR}

The interaction of electromagnetic waves with the human organism is highly sophisticated. In order to describe the level of interaction of the human organism with electromagnetic waves, in the 1860s, American scholar Schwan proposed the definition of specific absorption rates (SAR). SAR is usually used to describe the interaction of electromagnetic waves with human and identify the maximum limit level of electromagnetic radiation for human safety. SAR represents the time derivative of electromagnetic energy absorbed or consumed by a $1 \mathrm{~kg}$ mass of the human body. SAR has unit of $\mathrm{W} / \mathrm{Kg}$, which can be calculated as:

$$
\mathrm{SAR}=\frac{d}{d t}\left[\frac{d W}{d m}\right]=\frac{d}{d t}\left[\frac{d W}{\rho \cdot d V}\right]
$$

Where $t$ represents time; $W$ represents radiated power; $m$ represents the weight of biological tissue, $V$ represents the volume of biological tissues; $\rho$ represents the biological tissue conductivity. 


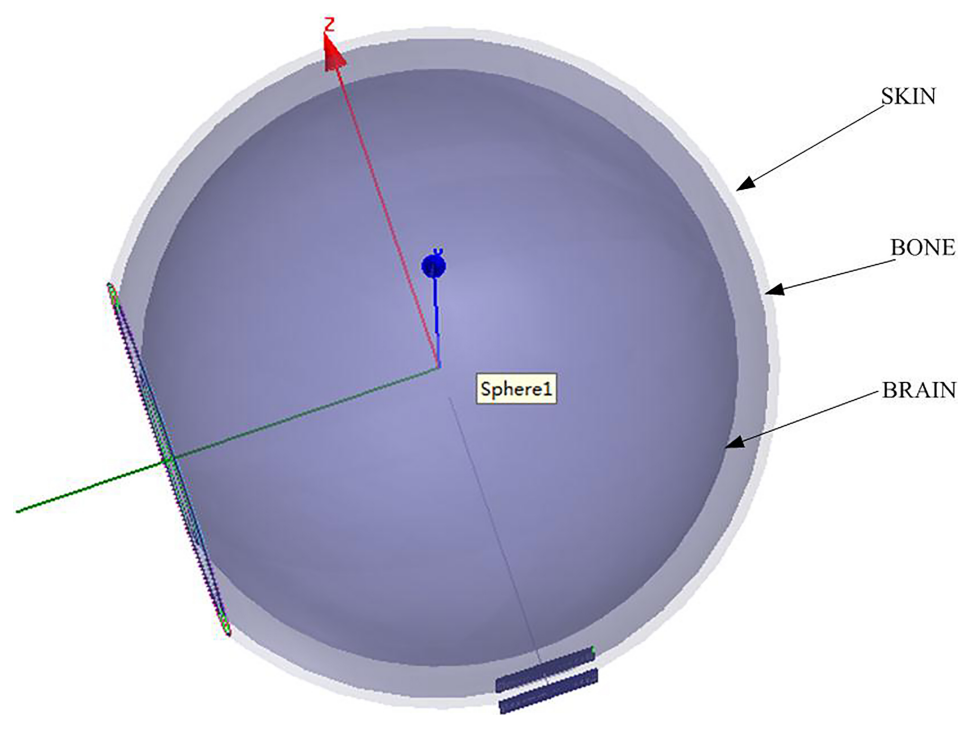

Fig. 4. Three-layer head model.

In theory, dielectric properties of different part of human tissues are discrepant, which led to the uneven distribution of electromagnetic field in human tissues. The level of electromagnetic radiation energy that human tissue absorbs is associated with the conductivity of the human organ. Most human tissue contains a lot of water, which leads to the high conductivity. Therefore, SAR of different parts of the body are different. However, there exists the need to describe the electromagnetic energy absorbed by the body in electromagnetic environment, which led people to put forward the concept of average SAR. The average SAR is calculated as follows:

$$
\begin{aligned}
\operatorname{SAR}_{a v}(i, j, k)= & \frac{1}{2 \sum_{i, j, k} \rho(i, j, k) \cdot V(i, j, k)}\left[\sum _ { i , j , k } \left(\sigma_{x}(i, j, k)\left|E_{x}(i, j, k)\right|^{2} V(i, j, k)\right.\right. \\
& \left.\left.+\sigma_{y}(i, j, k)\left|E_{y}(i, j, k)\right|^{2} \mathrm{~V}(i, j, k)+\sigma_{z}(i, j, k)\left|E_{z}(i, j, k)\right|^{2} \mathrm{~V}(i, j, k)\right)\right]
\end{aligned}
$$

$s_{x}(i, j, k), E_{x}(i, j, k), V(i, j, k)$ represent the conductivity of each point along the $x, y, z$ direction, and $\rho(i, j, k)$ represents the tissue density of each point.

\subsection{Simulation}

We use FDTD method to calculate SAR of tissues through finite element method (FEM) software. Usually, The FDTD method was a kind of time-dependent equations for Maxwell's equations. The simulation is divided into two parts. In one situation, both the transmitting coil and receiving coil work, most of the energy is effectively transferred and there is low electromagnetic radiation. In the other situation, only the transmitter coil works, which means that the receiver battery has been fully charged or the receive coil does not work normally. In this situation, most of the energy is converted into electromagnetic radiation.

In the early model of a human head, the common models for SAR measurement is a thin sphere containing fully brain-tissue-equivalent materials. In this paper, a three-layer head model is established which can model the real head as shown in Fig. 4. The new model is composed of three layers, which are skin, bone and brain tissues. The parameters area as follows: the radius of the head is $11 \mathrm{~cm}$ and 
Table 1

Parameters of each layer

\begin{tabular}{lccc}
\hline Name & Conductivity $(\mathrm{S} / \mathrm{m})$ & Relative permittivity & Density $\left(\mathrm{kg} / \mathrm{m}^{3}\right)$ \\
\hline kin & 0.4310 & 74.25 & 1125 \\
bone & 0.1283 & 35.66 & 1080 \\
Brain & 0.3169 & 73.62 & 1027 \\
\hline
\end{tabular}

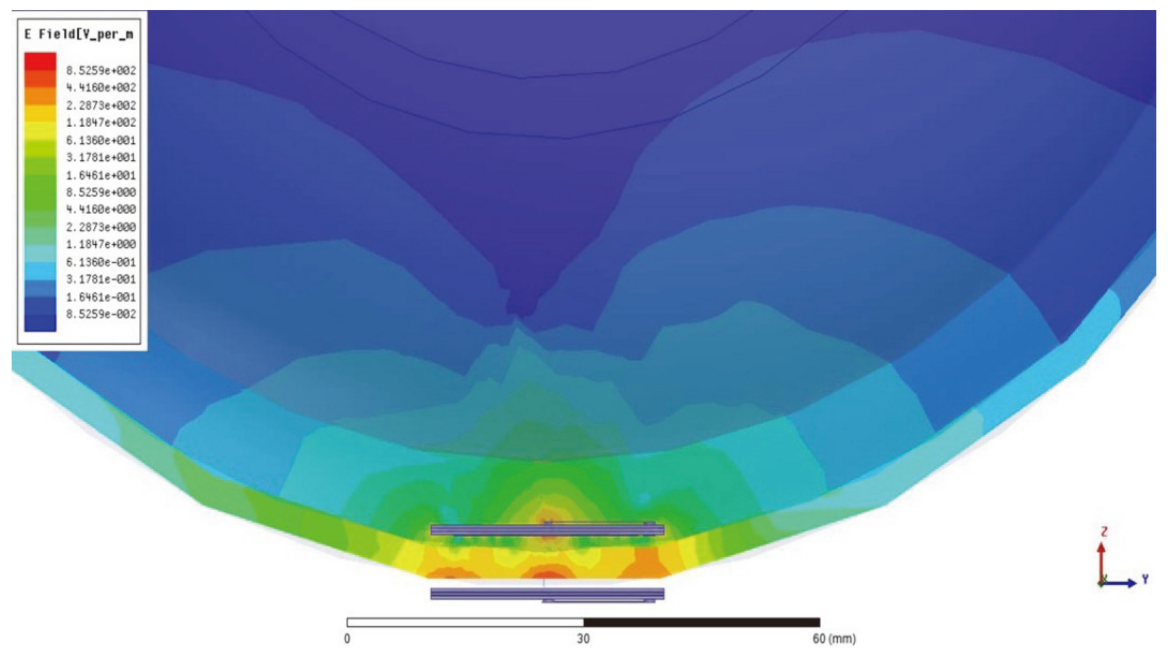

Fig. 5. Results of the situation both antenna works.

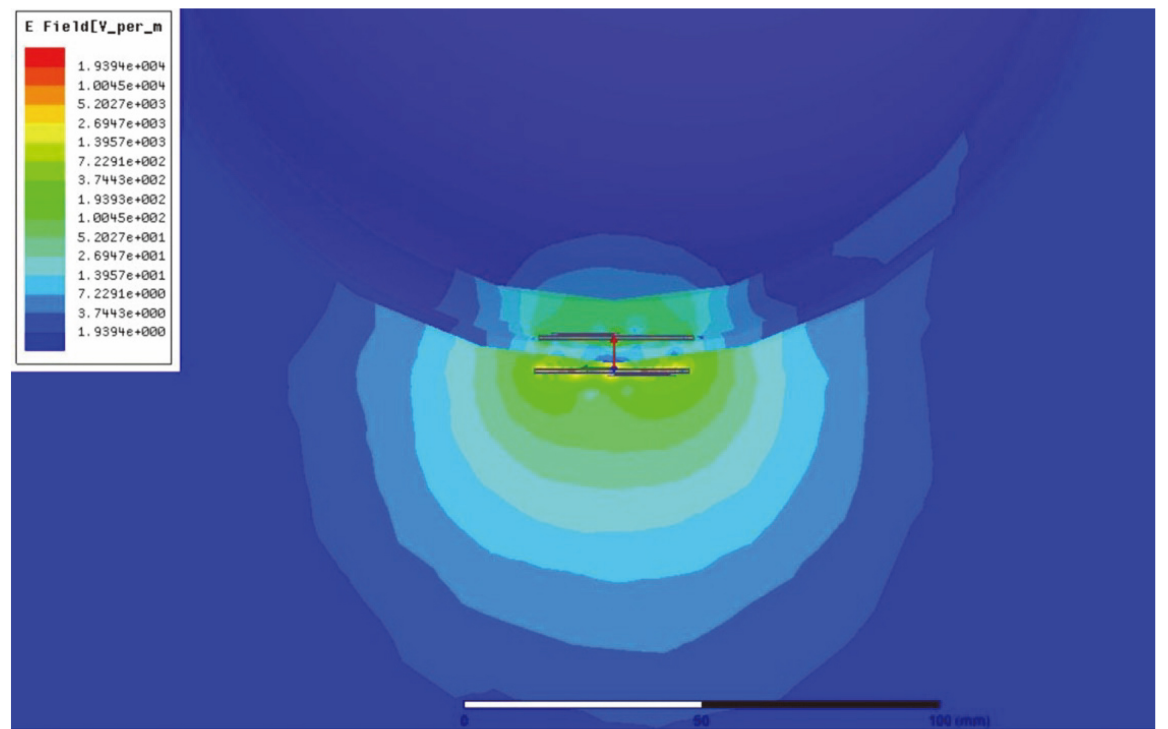

Fig. 6. E-field strength distribution when the system operating at $18.67 \mathrm{MHz}$.

the thickness of air layer is $1 \mathrm{~mm}$, the thickness of the skin layer is $3.5 \mathrm{~mm}$ and the thickness of bone is $10 \mathrm{~mm}$. The coil below is transmitting coil, and the above is accepting coil. The parameters of each layer are as shown in Table 1. 


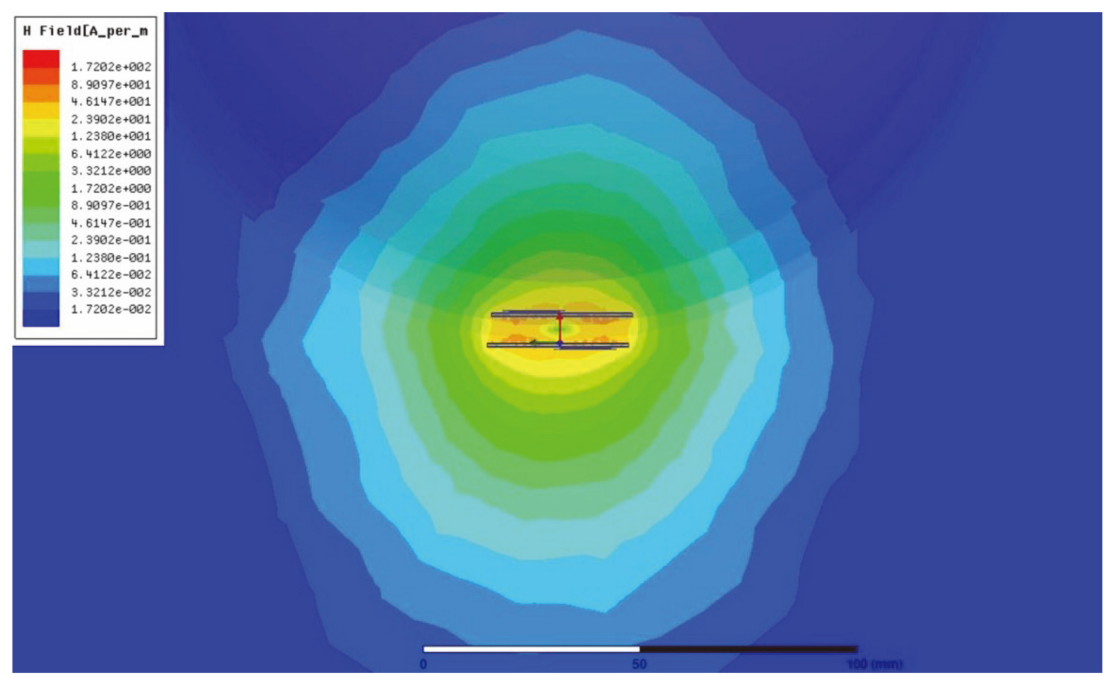

Fig. 7. M-field strength distribution when the system operating at $18.67 \mathrm{MHz}$.

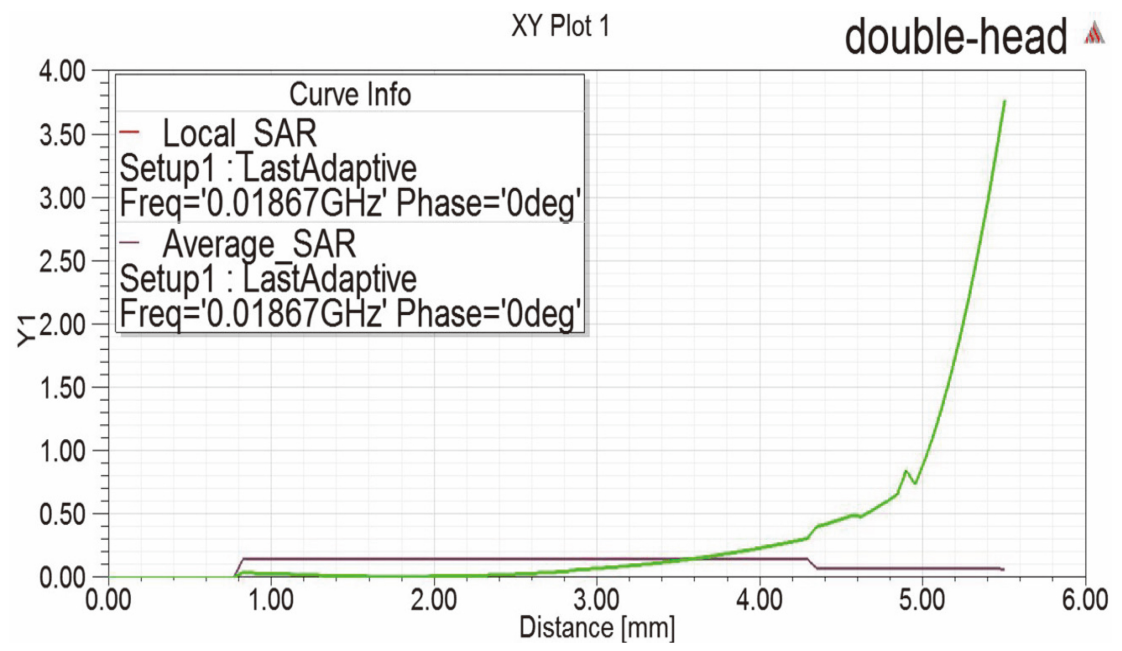

Fig. 8. SAR distribution in first positions.

\subsubsection{The first situation (both coils work)}

As mentioned in Chapter 3, the resonant frequency of the excitation is set to $18.67 \mathrm{MHz}$, the power is set to $1 \mathrm{~W}$. When the antenna system works in the resonance state, the simulation results are shown in Fig. 5, which shows that most of the power is effectively transferred. And most electromagnetic radiation appears in the middle of the two resonant coils. The distribution of electric field and magnetic field are as shown in Figs 6 and 7. The maximum of the electric field and magnetic field are respectively $1.94 \times$ $10^{4} \mathrm{~V}$ and $1.72 \times 10^{2} \mathrm{H}$.

In order to obtain data of radiation dose, the SAR values of two positions are calculated separately. One is between the coils, from the bottom to top, the other is from the upper receiver coil to the whole head. And the maximum of SAR are separately $0.142 \mathrm{~W} / \mathrm{Kg}$ and $0.059 \mathrm{~W} / \mathrm{Kg}$. 


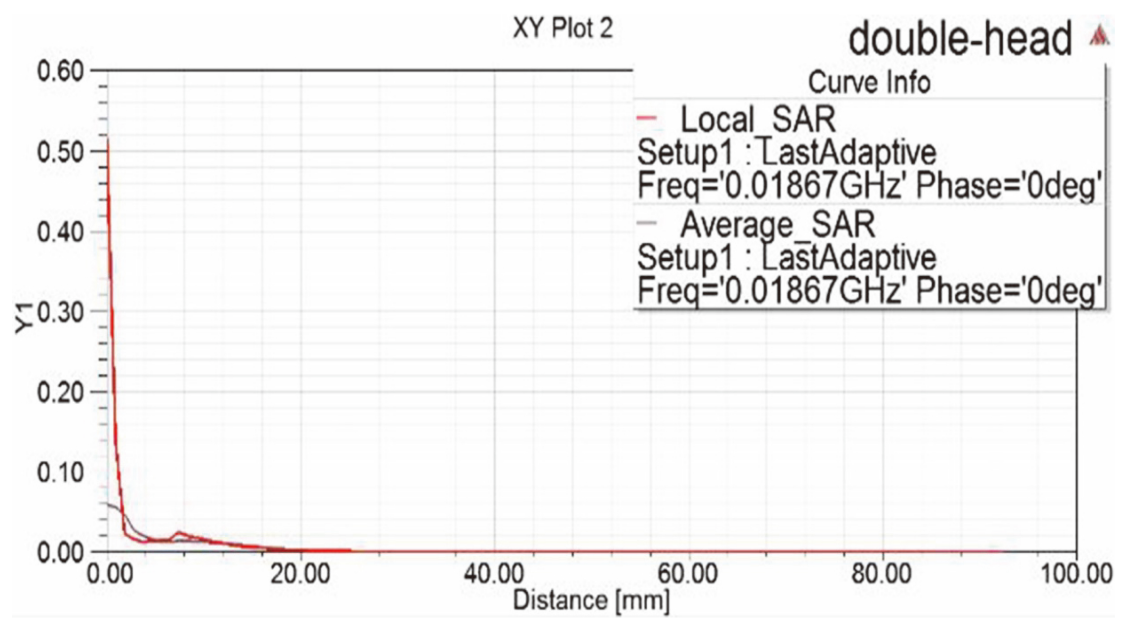

Fig. 9. SAR distribution in second positions.

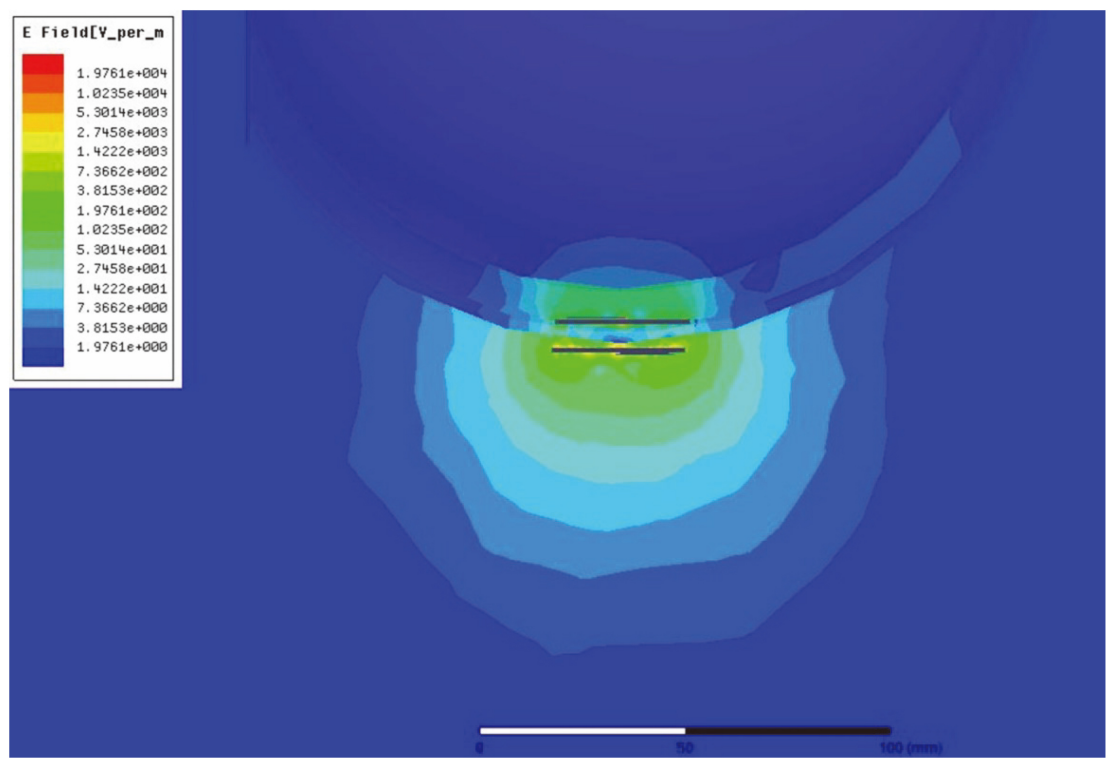

Fig. 10. E-field strength distribution when the system operating at $18.67 \mathrm{MHz}$.

\subsubsection{The second situation (only transmitting coil works)}

And in the second situation only the transmitting coil works, most of the power will be converted to be electromagnetic radiation. In this situation, the SAR value will be bigger than the first situation. We use the same parameters as shown in Section 4.2.1. The results are shown in Figs 10 and 11.

The distributions of electric field and magnetic field in the situation when only transmitting antenna works are as shown in Figs 11 and 12. The maximum of the electric field and magnetic field are respectively $1.97 \times 10^{4} \mathrm{~V}$ and $2.158 \times 10^{2} \mathrm{H}$, both are bigger than the first situation. And the maximum of SAR are separately $0.148 \mathrm{~W} / \mathrm{Kg}$ and $0.061 \mathrm{~W} / \mathrm{Kg}$.

As shown in Figs 8, 9, 12 and 13, SAR value between the two coils around the transmitting coil and receiving coil in both situations is significantly higher than the other place. SAR values of two posi- 


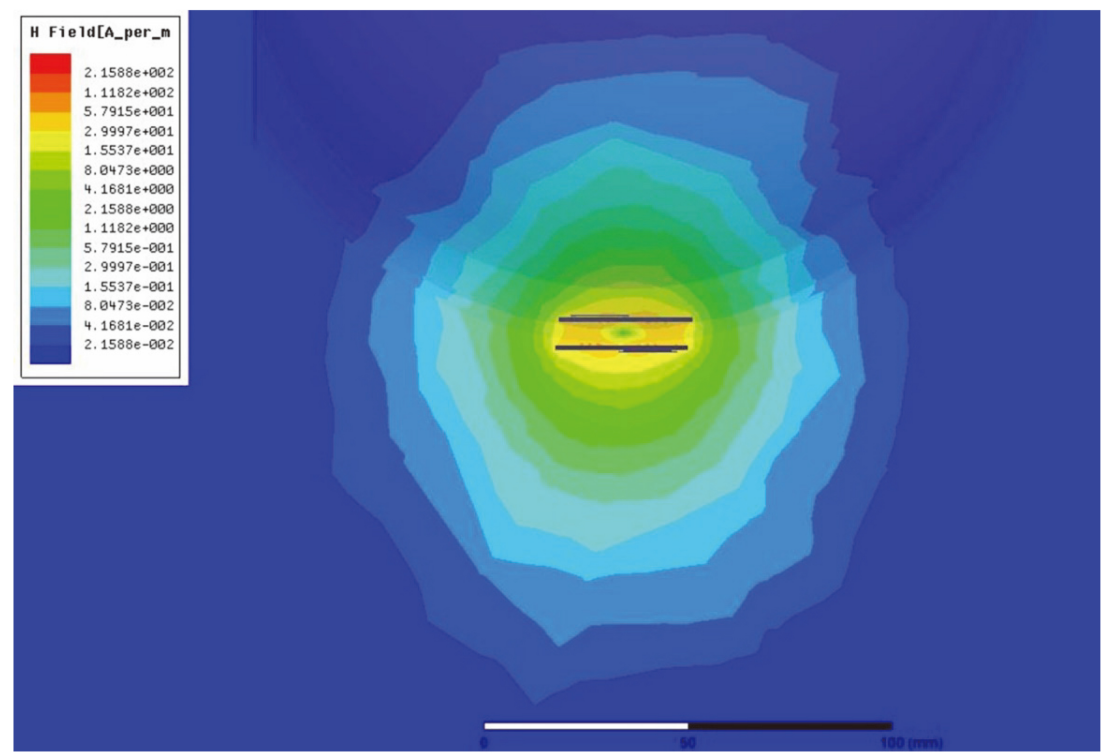

Fig. 11. M-field strength distribution when the system operating at $18.67 \mathrm{MHz}$.

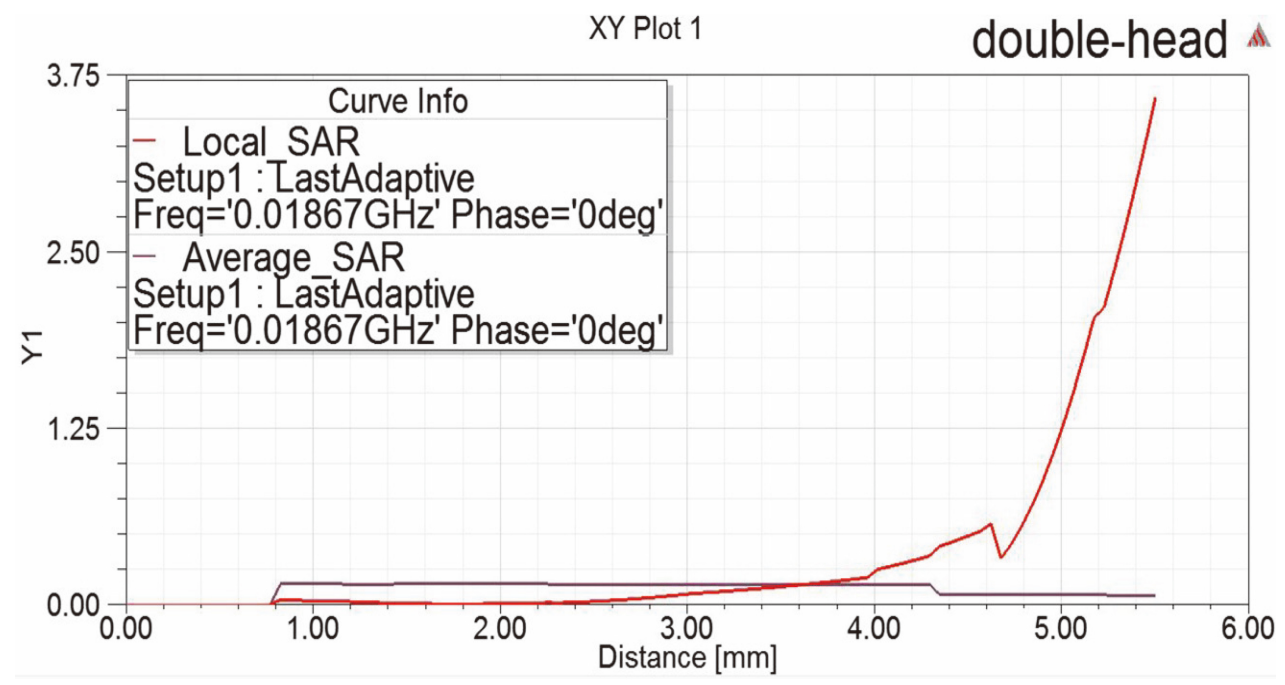

Fig. 12. SAR distribution in first positions.

tions in the second situation are higher than the first situation the reason is that only the transmitter coil works, most of the energy is converted into electromagnetic radiation. There are a universal electromagnetic radiation safety standards named International Commission on Non-Ionizing Radiation Protection (ICNIRP) and a national standard of America named Federal Communications Commission (FFC) standards both require that the SAR level is at or low $1.6 \mathrm{~W} / \mathrm{Kg}$ spread over a volume of $1 \mathrm{~g}$ of tissue, whereas the SAR limit is $2 \mathrm{~W} / \mathrm{kg}$ averaged over of $10 \mathrm{~g}$ of tissue. The maximum of all the SAR value is $0.148 \mathrm{~W} / \mathrm{kg}$ averaged over of $10 \mathrm{~g}$ of tissue which is lower than the limit of both standards. As a matter of fact, SAR is associated with the transmission path of the electromagnetic field. In the resonant state, 


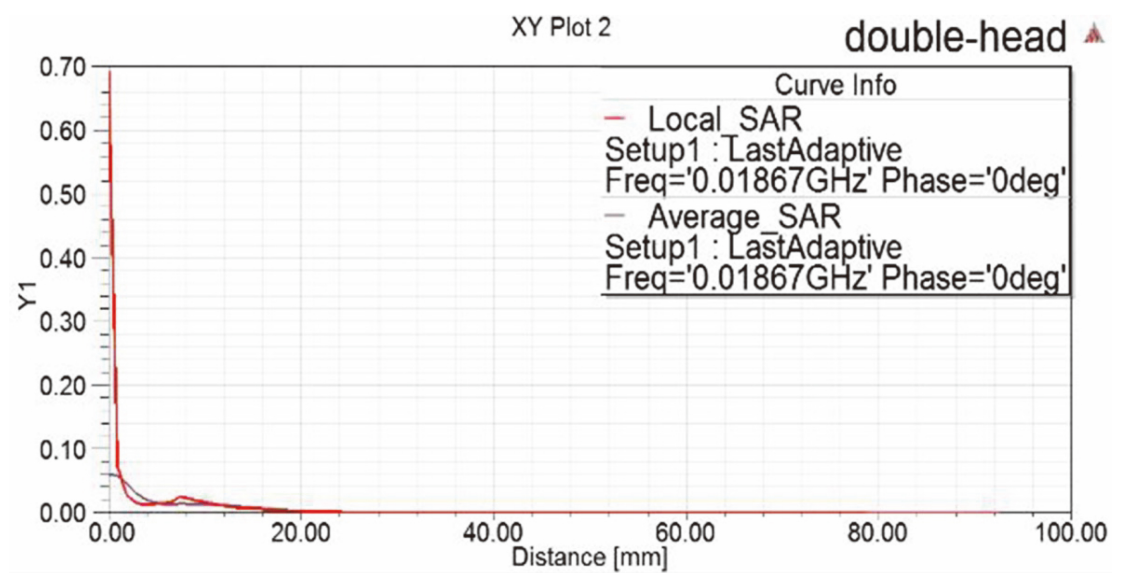

Fig. 13. SAR distribution in second positions.

most of the power was transferred along the path from the transmitting coil to the receiving coil, so the local and average value of SAR is bigger than the scenario that only the transmitting coil worked because the power was spread into the space in all directions.

\section{Conclusions}

In this paper, four-layer antenna system with epoxy resin glass fabrics (ERGB) between each couplelayer was designed for human implantable WPT system. The resonance frequency simulated by HFSS software was $18.67 \mathrm{MHz}$, and the Q factor was 62.23. A new head model composed of three layers was established, which were skin, bone and brain tissues. The antennas were situated inside and outside the head model at $6 \mathrm{~mm}$ distance. Both workstations were considered, in one situation both coils worked and in the other situation only one coil worked. The local and average SAR were simulated at the resonance frequency 18.67 MHZ. The results showed that the $10 \mathrm{~g}$ SAR average value of human thoracic were $0.142 \mathrm{~W} / \mathrm{kg}$ and $0.148 \mathrm{~W} / \mathrm{kg}$, respectively, which were lower than the safety standards of the ICNIRP and FCC, which verified the safety of the human body in wireless power transmission process based on magnetic coupling resonance.

\section{Acknowledgments}

This paper is supported by the National International Cooperation Projects "Joint Research and Development of Magnetic Resonance Wireless Energy Transfer System for Implantable Electronic Devices" (2013DFA10490) and Qingdao innovation and entrepreneurship leading project (13-cx-2).

\section{Conflict of interest}

None to report. 


\section{References}

[1] Kurs A, Karalis A, Moffatt R, Joannopoulos JD, Fisher P, and Soljačić M. Wireless power transfer via strongly coupled magnetic resonances, Science (80-.) vol. 317; no. 5834, pp. 83-86, 2007.

[2] Rubin GJ, Rw H, and Nieto S. Idiopathic environmental intolerance attributed to electromagnetic fields (formerly electromagnetic hypersensitivity): An updated systematic review of provocation studies, Bioelectromagnetics, vol. 31; no. 1, pp. C1-C11, 2010.

[3] Schiavoni A, Bertotto P, Richiardi G, and Bielli P. SAR generated by commercial cellular phones-phone modeling, head modeling, and measurements, Microw. Theory Tech. IEEE Trans., vol. 48; no. 11, pp. 2064-2071, 2000.

[4] Sullivan DM, A frequency-dependent FDTD method for biological applications, Microw. Theory Tech. IEEE Trans., vol. 40; no. 3, pp. 532-539, 1992.

[5] Shiba K, Nagato T, Tsuji T, and Koshiji K. Energy Transmission Transformer For A Wireless Capsule Endoscope: Analysis Of Specific Absorption Rate And Current Density In Biological Tissue, Biomed Eng. IEEE Trans, vol. 55; no. 7, pp. 1864-1871, 2008.

[6] Liang A, Research on electromagnetic dosimetry for variable posture of human body model, Xian university of electronic science and technology, 2006.

[7] Liu C, Numerical simulation research on human head SAR, Beijing university of posts and telecommunications, 2010.

[8] Park JD, Kim BC, and Choi HD. Analysis of SAR Caused by PIFA Mounted on Folder type Mobile Phones, Radio\&Broadcasting Technol. Lab. ETRI, 2002.

[9] Neubauer G, Feychting M, Hamnerius Y, Kheifets L, Kuster N, Ruiz I, Schüz J, Überbacher R, Wiart J, and Röösli M. Feasibility of future epidemiological studies on possible health effects of mobile phone base stations, Bioelectromagnetics, vol. 28; no. 3, pp. 224-230, 2007.

[10] Choi D, Shin C, Kim N, and Shin H. Design and SAR analysis of broadband PIFA with triple band, PIERS Online, vol. 1; no. 3, pp. 290-293, 2005.

[11] Christ A, Douglas M, Nadakuduti J, and Kuster N. Assessing Human Exposure to Electromagnetic Fields From Wireless Power Transmission Systems, Proc. IEEE, vol. 101; no. 6, pp. 1482-1493, 2013.

[12] Xin WH, Yan GZ, Wang WX, and Jia ZW. Study on stability of power receiving for capsule endoscopes [J], Chinese J. Sci. Instrum. vol. 11; p. 34, 2009.

[13] Zhao GXJ, Research on the effects of electromagnetic radiation to human body by impantable cois basen on resonant wireless power transmission system, Chinese J. Biomed. Eng, vol. 31; no. 5, pp. 649-654, 2012.

[14] Lak A, and Oraizi H. Evaluation of SAR Distribution in Six-Layer Human Head Model, Int. J. Antennas Propag, vol. 12; no. 1, pp. 56-64, 2013.

[15] Liu X, Zhang F, Hackworth S, Sclabassi RJ and Sun M. Wireless power transfer system design for implanted and worn devices, in Bioengineering Conference, 2009 IEEE 35th Annual Northeast, , pp. 1-2, 2009. 